\title{
Hands-Free Wearable Electrolarynx using Linear Predictive Coding Residual Waves and Listening Evaluation
}

\author{
Masaki TAKeuchi, ${ }^{*}$ \# Jaesol Ahn, ${ }^{* *}$ Kunhak LeE, ${ }^{* * *}$ Ken TAKaKI, ${ }^{*}$ Tohru Ifukube, ${ }^{\dagger}$ Ken-ichiro Yabu, ${ }^{\dagger}$ \\ Shinnosuke TAKAMICHI, ${ }^{\dagger \dagger}$ Rumi UeHa, ${ }^{\dagger \dagger}$ Masaki SEKINO ${ }^{\ddagger}$
}

\begin{abstract}
A conventional electrolarynx (EL), which is used by laryngectomees, produces monotonous sound and occupies a user's hand; hence, we developed a hands-free wearable device that improves voice quality. The proposed device estimates individual vocal tract features using linear predictive coding (LPC) and generates sound vibrations using an LPC inverse filter. Additionally, we reproduced the vibration sound using a transducer and amplified the first harmonic frequency and the second one. We conducted an objective experiment to compare the spectra of natural voice, a conventional EL, and the proposed device. We also conducted a subjective experiment in which we asked healthy subjects to listen to and evaluate the conventional EL and the proposed device. The results of the objective experiment demonstrated that our model was characterized by two formant peaks that were similar to the conventional EL and the natural voice. The results of the subjective experiment demonstrated that our model was more powerful and clearer than the conventional EL. These findings indicate that the voice of our device is spectrally close to human voice and gives the audience a more powerful and clearer sound.
\end{abstract}

Keywords: welfare device, signal processing, transducer.

Adv Biomed Eng. 11: pp. 68-75, 2022.

\footnotetext{
This study was presented at the Symposium on Biomedical Engineering 2021, September, 2021.

Received on July 26, 2021; revised on October 31, 2021 and January 9, 2022; accepted on January 20, 2022.

* Graduate School of Engineering, The University of Tokyo, Tokyo, Japan.

** Faculty of Information and Communication Engineering, The University of Tokyo, Tokyo, Japan.

${ }^{* * * *}$ Faculty of Mechanical Engineering, The University of Tokyo, Tokyo, Japan.

${ }^{\dagger}$ Research Center for Advanced Science and Technology, The University of Tokyo, Tokyo, Japan.

${ }_{\dagger \dagger}$ Department of Information Science and Technology, The University of Tokyo, Tokyo, Japan.

${ }^{\dagger \dagger}$ Swallowing Center, The University of Tokyo Hospital, The University of Tokyo, Tokyo, Japan.

${ }^{\ddagger}$ Department of Bioengineering, The University of Tokyo, Tokyo, Japan.

\# 2-11-16 Yayoi, Bunkyo-ku, Tokyo 113-0032, Japan.

E-mail: takeuchi-masaki854@g.ecc.u-tokyo.ac.jp
}

\section{(cc) BY}

Copyright: (02022 The Author(s). This is an open access article distributed under the terms of the Creative Commons BY 4.0 International (Attribution) License (https:// creativecommons.org/licenses/by/4.0/legalcode), which permits the unrestricted distribution, reproduction and use of the article provided the original source and authors are credited.

\section{Introduction}

People typically communicate using their voices. The human voice is produced as follows. First, exhaled air flows from the lungs to the larynx. Thereafter, the mucous membrane of the vocal cords repeatedly opens and closes at high speed, further causing the airflow from the lungs to vibrate and produce sound. Finally, owing to the differences in the shapes of the mouth and tongue, the resonance frequency in the vocal tract varies, further creating different types of sound, thus producing the voice. However, people whose vocal cords have been removed because of laryngeal cancer cannot send air to the vocal cords, and consequently are not capable of producing sound. Hence, they have speech impediments, because although they can move their mouth, they are unable to produce sound.

One way by which such people can regain their voice is by using an electrolarynx (EL). An EL user presses a button on a cylindrical machine to generate electrical vibration. However, the user cannot speak unless he/she holds the EL with one hand and presses it against the throat. Vibrations of only a single frequency can be generated, and the sound will be monotonous and mechanical, regardless of the user.

A few researchers have attempted to address these 
challenges by developing a hands-free EL. Uemi et al. [1] developed a hands-free pitch-control EL based on the expiration pressure. Hashiba et al. [2, 3] developed and evaluated a wearable EL equipped with a wireless on/off switch and pocket-sized controller. Although a hands-free model could be realized, its voice quality and usability, which are important for users, were mediocre. Matsui et al. [4] developed a hands-free EL using a micro-electromechanical system accelerometer to control the pitch via arm movement. Two types of pitch contour generation methods were proposed and tested along with a conventional EL. However, the vibration sound was similar to a robotic sound, and people without the ability to control their arms could not use it.

Other hands-free ELs include electromyography (EMG) sensors. Goldstein et al. [5] first designed and implemented a hands-free EL that could be controlled using EMG. Subsequently, some researchers developed a hands-free EL using neck and face surface EMG (sEMG) to control voice initiation, voice termination, and pitch [6-9]. However, these studies did not realize a natural voice.

Moreover, several studies have been conducted on improving the sound quality using machine learning [10-15], noise reduction [16-20], or both [21]. Although these systems improve the intelligibility of the output voice, the speaker outputs the voice. Thus, users cannot generate syllables from their mouths. This causes a delay between the movement of the mouth and the generation of voice.

Accordingly, this study aims to develop a novel hands-free EL (Fig. 1) for voice restoration with sound vibrations similar to those of the human voice. Moreover, we evaluated the output voice of our device by spectral comparison and voice listening experiments.

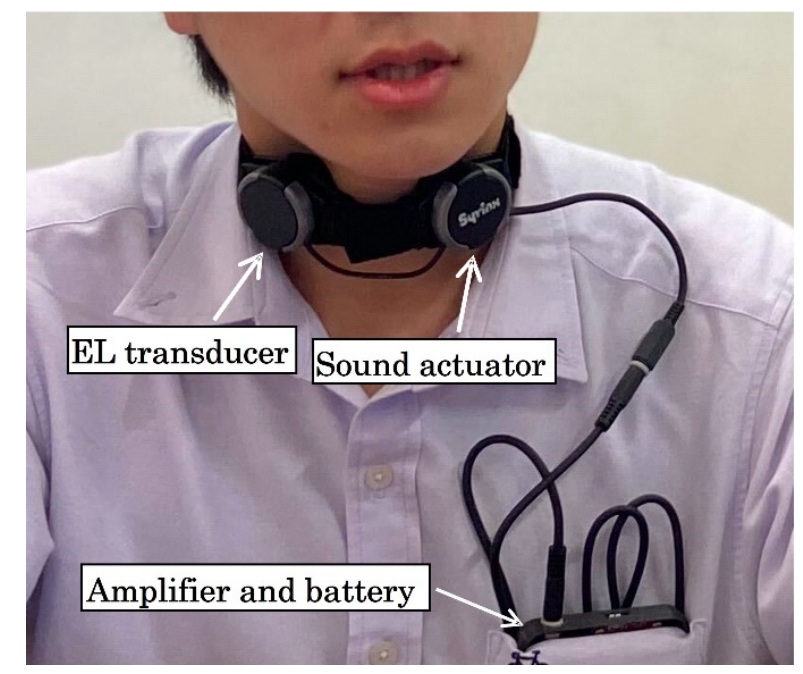

Fig. 1 Photograph of a person using the proposed device.

\section{Materials and Methods}

\subsection{Device structure}

Figure 2 depicts the proposed system. The sound vibrations, which are generated from a computer or smartphone, are sent to transducers via Bluetooth and a cable. The proposed device had two cylindrical transducers that were placed on the throat while speaking. The device vibrated the throat to produce natural sounds through the vocal tract. To generate a natural sound, we estimated sound vibrations from raw voice in advance, as described in Section 2.2. We also used an EL transducer and a sound actuator. The EL transducer was a hitting-type transducer, and was used in a conventional EL. The sound actuator was the ACOUSTICHAPTIC ${ }^{\circledR}$ Actuator, LA-0031, $\varphi 20 \times 30$, provided by Foster Electric Co., Ltd. We used two different types of transducers to achieve a wider frequency range and louder volume. The EL transducer had a narrower frequency range of $60-200 \mathrm{~Hz}$, but its output volume was louder. However, the output volume of the sound actuator was lower than that of the EL transducer. Two different types of vibrators were needed to produce sounds for both men and women of all ages, as the EL transducer had a narrower frequency range but greater vibration, while the sound actuator had a wider frequency range but smaller vibration.

\subsection{Generation of natural sound vibrations}

We used linear predictive coding (LPC) to estimate the sound vibrations. LPC assists in extracting parameters of vocal tract features [22]. LPC residue is one of the major driving signals in source-filtered speech synthesis. In fact, some studies have demonstrated that it can produce vowels in a manner similar to that of the human voice when applied to the EL [23]. To acquire the prediction function for minimizing the difference between the predicted and actual values, a linear combination of values obtained from previous samples was used as the predict-

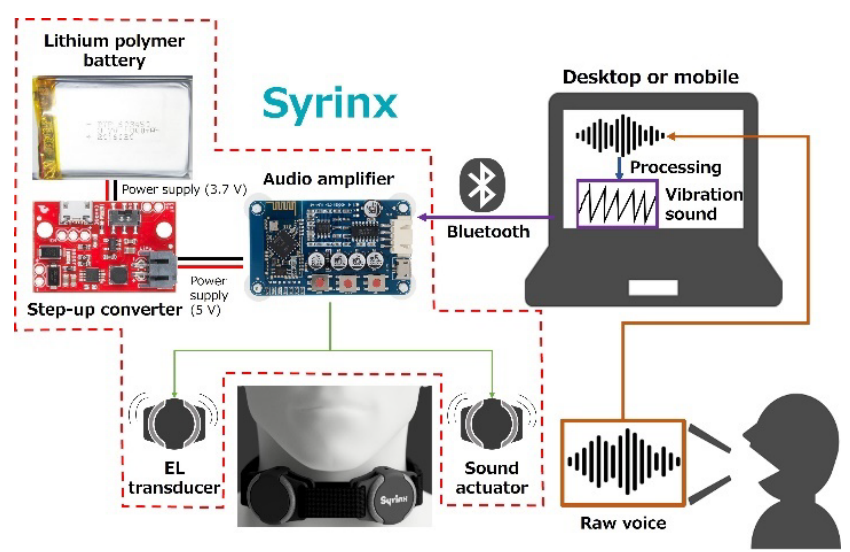

Fig. 2 System design. 
ed value, which is denoted by $x_{n}$, and is expressed as follows:

$$
x_{n}=-\left(a_{1} s_{n-1}+a_{2} s_{n-2}+\ldots+a_{p} s_{n-p}\right),
$$

where $a_{n}$ is the linear predictor coefficient, and $p$ is the LPC order. $s_{n}$ is the actual recorded data at time $n$, and $e_{n}$ is the prediction error within the sampling interval. $a_{n}$ was calculated to minimize the squared value of $e_{n}$, which is expressed as

$$
e_{n}=s_{n}+a_{1} s_{n-1}+a_{2} s_{n-2}+\ldots+a_{p} s_{n-p} \text {. }
$$

Thereafter, Eq. (2) was $z$-transformed as follows:

$$
\begin{aligned}
& E(z)=S(z)\left(1+a_{1} z^{-1}+\ldots+a_{p} z^{-p}\right) \\
& S(z)=\frac{1}{1+a_{1} z^{-1}+\ldots+a_{p} z^{-p}} E(z) \\
& S(z)=H(z) E(z)\left(\because H(z)=\frac{1}{1+a_{1} z^{-1}+\ldots+a_{p} z^{-p}}\right) \\
& E(z)=\frac{1}{H(z)} S(z) .
\end{aligned}
$$

$\frac{1}{H(z)}$ indicates an LPC inverse filter. The vocal tract parameters can be removed by the human voice by multiplying the LPC inverse filter. This results in LPC residual waves. We recorded two voices to estimate the actual LPC residual wave: a voice generated with the transducer and a human voice with normal tone. A chirp signal, which is typically used to measure the frequency characteristics, was used for speaking with the transducer. The user opened the mouth in a manner that pronounced the Japanese vowel /a/ and placed the transducer that generated the chirp signal on the throat. The recording duration was $5 \mathrm{~s}$. Let $X(z)$ and $Y(z)$ be the z-transforms of the chirp signal and the signal output from the mouth, respectively, then $H(z)$ can be expressed as

$$
H(z)=\frac{Y(z)}{X(z)} .
$$

To record a normal tone, the user said /a/ using the vocal cord. Let $Y^{\prime}(z)$ be the z-transforms of the output voice and LPC residual wave, then $X^{\prime}(z)$ can be expressed as

$$
X^{\prime}(z)=\frac{Y^{\prime}(z)}{H(z)} .
$$

Subsequently, we calculated the LPC residual wave, as shown in Fig. 3. Unlike the waves generated by the human voice, the sound pressure levels of the first harmonic frequency and the second harmonic are less than approximately $20 \mathrm{~dB}$ (Fig. 3b).

Therefore, using an audio equalizer in Audacity, we increased the levels of the first harmonic frequency and second harmonic in the output of our device to obtain amplitudes similar to the human voice waves. The first harmonic frequency is the smallest periodic interval of a periodic speech signal. If the voice waveform is enlarged, (a)
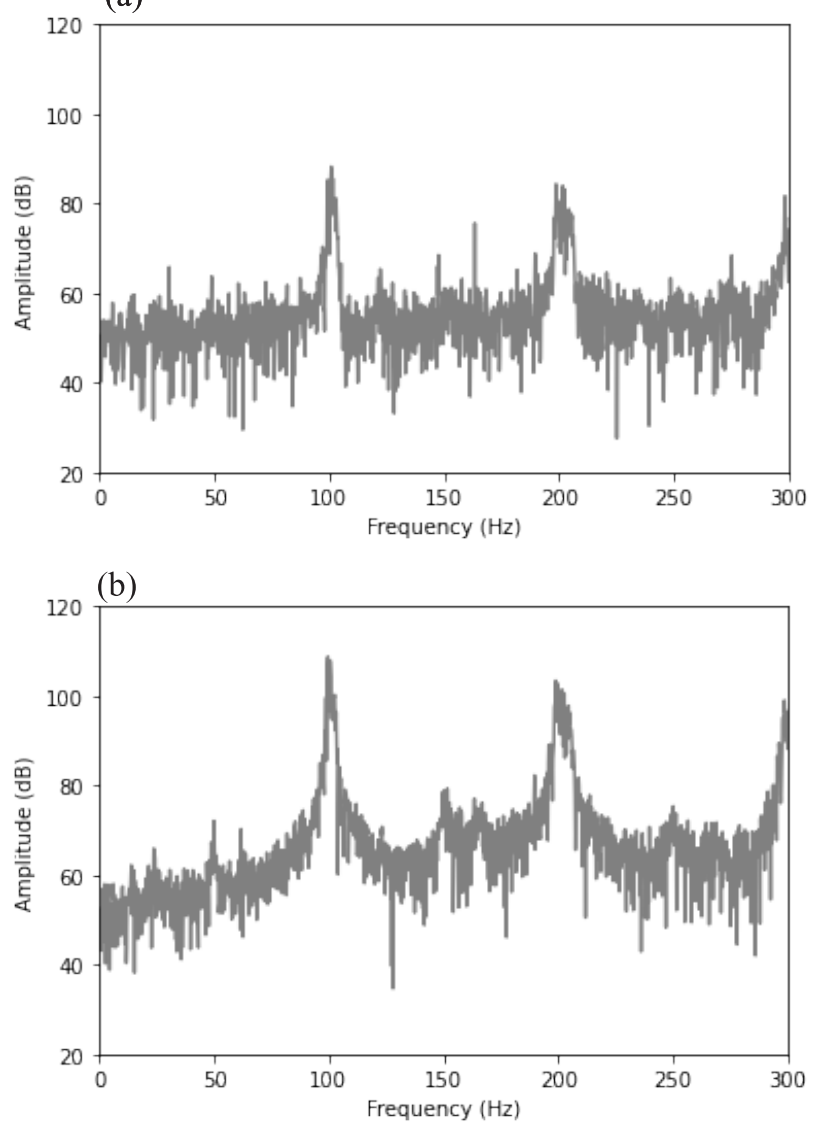

Fig. 3 Frequency spectrum of (a) a voice generated with LPC and (b) of a human voice.

the same waveform is repeated constantly. When repeated every $0.01 \mathrm{~s}$, it is repeated 100 times in $1 \mathrm{~s}$. In other words, the first harmonic frequency is $100 \mathrm{~Hz}$. The first harmonic frequency is also known as F0. In this case, the first harmonic frequency is $100 \mathrm{~Hz}$. The second harmonic is twice the value of the first harmonic frequency.

\subsection{Spectrum comparison}

We compared the frequency spectra of our device, the conventional EL, and natural voice, without any change in the first harmonic frequency (F0). This is the pitch of the voice. All voices were produced by the lead author of this paper. He is not a laryngectomee. However, there is no significant difference in the shape of the vocal tract between laryngectomees and non-laryngectomees. Hence, no difference in the test results is expected. The recording environment comprised a PC (Panasonic, denoted as CF-5V hereinafter), a microphone (Audiotechnica, ATR1100x), a USB audio conversion adapter (Planex, PL-US35AP), and Praat, which is the application software used to record and conduct the listening evaluation experiments [24]. The recorded phrase was "Konnichiwa," which means "good afternoon" in Japanese. We extracted the vowel "a" for $0.06 \mathrm{~s}$ and Fourier- 
transformed the signals to display the frequency spectrum.

\subsection{Listening evaluation}

Eight subjects aged between 20 and 40 years with normal hearing participated in this study. They listened to the voice as two different types of speech (generated with a conventional EL and with the proposed device) and a total of 20 ATR sentences, ten each for the conventional EL and the proposed device. The A-weighted filtering sound levels were unified using MATLAB. A-weighted filtering is a sound pressure level used as a noise loudness measure, similar to human hearing [25]. We used the Praat Experiment MFC to conduct a multiple forced choice listening experiment for the listening evaluation. The order of the listening sounds was randomized. Our experiment used the SD method, which was used in previous studies as a subjective method to evaluate the substitute vocalization of laryngectomized patients [26, 27]. The SD method is a 7-point grading method for two adjectives A and B with polar meanings as follows: 1) very likely A; 2) likely A; 3) fairly likely A; 4) neither likely A nor likely B; 5) fairly likely B; 6) likely B; and 7) very likely B. The scales used were selected from a previous study [27], but we extracted the voice quality scales only, and eliminated scales for tempo and pitch. There was no breathing involved in this vocalization, therefore "breathy - not breathy" was excluded. We also excluded "slack - firm," which is not a good scale for comparison of voice quality. Then, we added scales related to naturalness [28], individuality [29], and listening effort [30], which are subjective scales commonly used in the speech synthesis field and research on substitute vocalization in laryngectomized patients. Thus, the list of scales is as shown in Table 1.

In this experiment, the lead author of this paper read ATR sentences using the conventional EL and the proposed device. ATR sentences are phonetically balanced

Table 1 List of scales used in this study.

\begin{tabular}{lll}
\hline \hline number & negative & positive \\
\hline 1 & ugly & beautiful \\
2 & not intelligible & intelligible \\
\hline \hline 3 & weak & powerful \\
\hline \hline 4 & expressionless & expressive \\
\hline \hline 5 & melodious & monotonous \\
\hline \hline 6 & dull & clear \\
\hline \hline 7 & unnatural & natural \\
\hline \hline 8 & not individual & individual \\
\hline \hline 9 & bad listening effort & good listening effort \\
\hline \hline
\end{tabular}

sentences extracted from newspapers, journals, novels, letters, and textbooks, among others, such that several phonetic environments occur at the same rate as much as possible [31]. We used 20 ATR sentences from A31A50. Two experimental sets were created and designated $\mathrm{A}$ and B. Set A included 10 sentences (A31-A40) spoken with the conventional EL and 10 sentences (A41A50) spoken with the proposed device. Set B was vice versa. All eight subjects participated in the experiment, four in each set. The experimental data were analyzed by Shapiro-Wilk test. As the experimental data were not normally distributed, Mann-Whitney $U$ test was used to determine whether there was a significant difference between the conventional EL and the proposed device for each scale, using $\mathrm{R}$ version 4.0.3. Statistical significance was set at $p<0.05$. We divided the experiments into practice and test sessions. All procedures performed in this study were in accordance with the ethical standards of the Ethical Review Committee of the University of Tokyo. All participants provided written informed consent.

\section{Results and Discussion}

To investigate the formants of the methods, we measured their spectra. A formant is a peak in the spectral envelope produced by the resonance of the vocal tract. Figure 4 shows the spectra of the natural voice, the EL output voice, and the output voice produced by the proposed device, respectively. Figure 4a shows the peaks at the first formant, F1 $(790 \mathrm{~Hz})$, and at the second formant, F2 $(1,190 \mathrm{~Hz})$. Fig. $4 \mathbf{b}$ and $4 \mathbf{c}$ show that the EL and the proposed device output specify peaks at F1 $(790 \mathrm{~Hz})$ and F2 $(1,190 \mathrm{~Hz})$, which represent the natural voice.

As depicted in Fig. 4c, our device reproduced the third formant (F3) at $2,700 \mathrm{~Hz}$, and the fourth formant (F4) at approximately $3,500 \mathrm{~Hz}$. F3 and F4 are related to personality and sound quality, respectively [22]. However, the proposed device did not reproduce the fifth formant (F5) at approximately 6,000 Hz. As depicted in Figs. $4 \mathbf{b}$ and $\mathbf{c}$, the frequency spectra of the EL and the proposed device did not show a clear formant after 4,000 Hz. Conversely, Fig. 4a shows that natural speech had a clear formant after $4,000 \mathrm{~Hz}$. This is due to mechanical impedance mismatch, which causes reflection from the oscillator to the oral cavity. The mechanical impedance of the skin present in the oral cavity of humans acts as a low-pass filter, which filters sound sources of $4,000 \mathrm{~Hz}$ and above [32]. This is presumably why F5 at $6,000 \mathrm{~Hz}$ could not be reproduced.

Figure 5 depicts the evaluation results of the conventional EL and the proposed device for each scale. These results suggest that there were significant differences with respect to "beautiful" ( $p<0.05)$, "clear" $(p<$ 
(a)

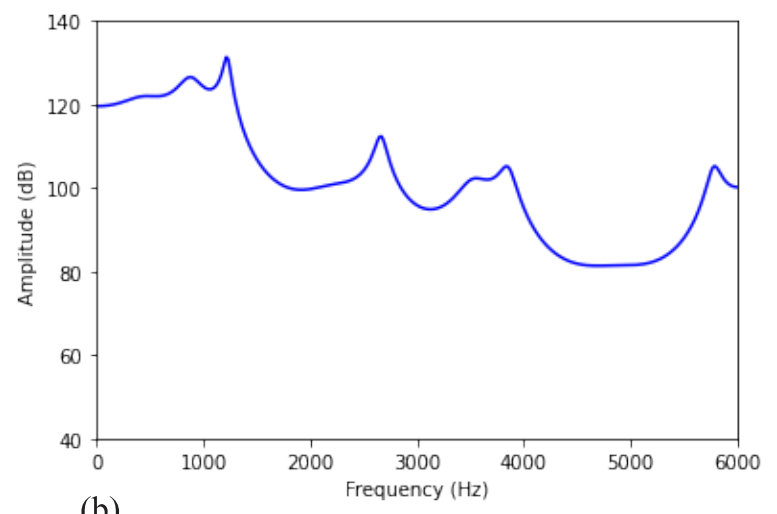

(b)

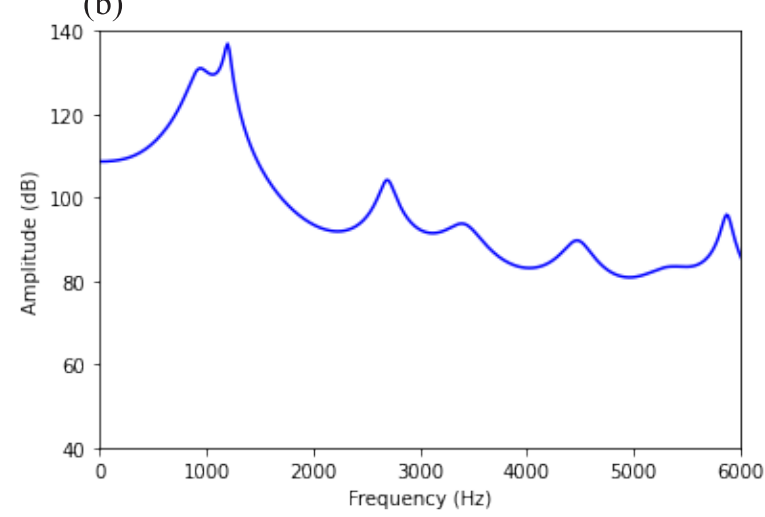

(c)

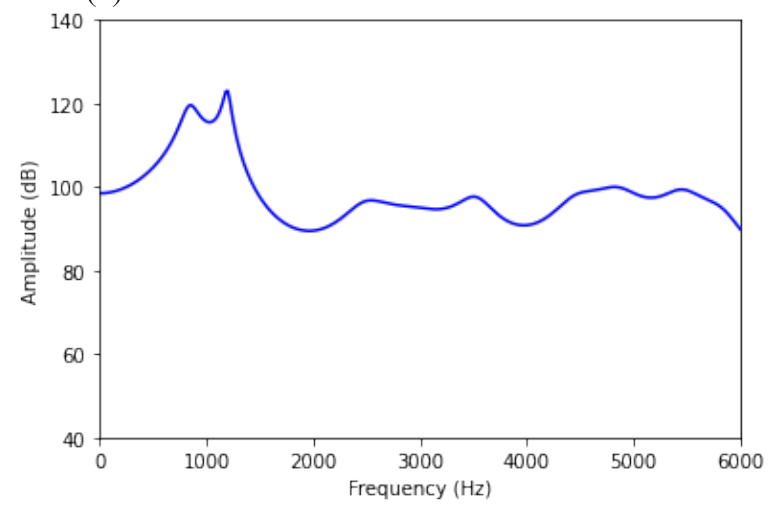

Fig. 4 (a) Natural voice, (b) EL output voice, and (c) proposed device voice for the Japanese vowel "a"; LPC spectral envelope of $0-6,000 \mathrm{~Hz}$.

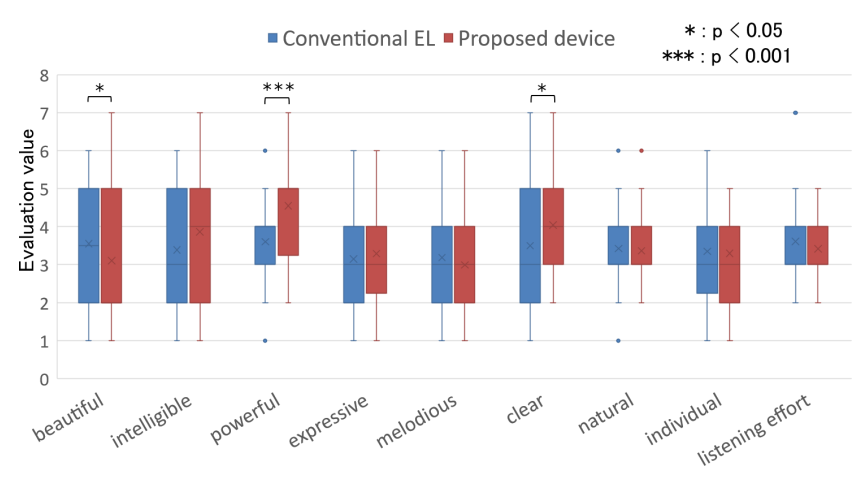

Fig. 5 Evaluation results of the experiment.
$0.05)$ and "powerful" $(p<0.001)$. For the "beautiful" scale, the conventional EL performs better than the proposed device. For the "clear" and "powerful" scales, the proposed device performs better than the conventional EL.

Significantly, the sound produced by our device was not more beautiful than that of the conventional EL. One participant claimed that he heard noises similar to the sound of a buzzer. The vibration sound from the transducer could not move efficiently through the neck surface. The reflected sound was the noise of the vibration sound from the transducer. This noise is the main reason for the difficulty in generating a beautiful output voice using the proposed device.

In future work, we will attempt to attenuate the vibration noise from the transducer. Moreover, a method to improve the vibration sound using an LPC residual wave was designed specifically for the lead author's voice. The validity of the proposed device must also be examined in other people.

\section{Conclusion}

In this study, we developed a device that assists laryngectomees to speak from the mouth, aiming to achieve sound vibrations similar to those of the human voice. We developed a novel hands-free device and generated sound vibrations using LPC residual waves. We conducted an objective spectral evaluation, which demonstrated that the proposed device produced a voice with characteristics similar to those of the natural voice and the conventional EL at 0-2,000 Hz. Furthermore, F3 and F4, which mainly reflect individuality, were superimposed on the artificial laryngeal voice. We verified the possibility of evaluating nine bipolar scales using the SD method. The results of listening evaluation demonstrated that the proposed device was more powerful and clearer than the conventional EL. Conversely, the sound produced by the conventional EL was more beautiful than that produced by the proposed device.

\section{Conflicts of interests}

The authors declare no conflicts of interest with any companies or commercial organizations according to the definition of the Japanese Society for Medical and Biological Engineering.

\section{Ethics declaration}

This study was conducted in accordance with the ethical principles of human subject research, and informed consent was obtained from each subject. The study was approved by the University-wide Ethics Review Committee of the University of Tokyo. The authorization number is KE21-70. 


\section{Acknowledgments}

We would like to express our sincere gratitude to Haruhiko Takemura of Foster Electric Co., Ltd., for providing the sound actuator. We are grateful to Yusuke Ito of RION Co., Ltd. for his help and support in conducting the listening evaluation. We are also grateful to Yutaro Soejima, a Bachelor's student at the University of Tokyo, for calculating the spectra. We would like to thank Editage (www.editage.com) for English language editing.

\section{References}

1. Uemi N, Ifukube T, Takahashi M, Matsushima J: Design of a new electrolarynx having a pitch control function. Proc of the IEEE International Workshop on Robot and Human Communication, Nagoya, pp. 198-203, 1994.

2. Hashiba M, Sugai Y, Izumi T, Ino S, Ifukube T: Development of a wearable electro-larynx for laryngectomees and its evaluation. Proc of the Annual International Conference of the IEEE Engineering in Medicine and Biology, Lyon, pp. 5267-5270, 2007.

3. Hashiba M, Sugai Y, T. Ifukube: OS3-2 commercialization of the multi-functional electro-larynx YOUR TONE II and it's further development for hands-free operation (OS3: Rehabilitation Devices I). Proc of the Asian Pacific Conference on Biomechanics: Emerging Science and Technology in Biomechanics, Sapporo, pp. 81, 2015.

4. Matsui K, Kimura K, Nakatoh Y, Kato YO: Development of electrolarynx with hands-free prosody control. Proc of the 8th ISCA Workshop on Speech Synthesis (SSW), Barcelona, Vol. 121, pp. 273-277, 2013.

5. Goldstein EA, Heaton JT, Kobler JB, Stanley GB, Hillman RE: Design and implementation of a hands-free electrolarynx device controlled by neck strap muscle electromyographic activity. Proc of the International IEEE/EMBS Conference on Neural Engineering, Capri, pp. 169-172, 2003.

6. Heaton JT, Goldstein EA, Kobler JB, Zeitels SM, Randolph GW, Walsh MJ, Gooey JE, Hillman RE: Surface electromyographic activity in total laryngectomy patients following laryngeal nerve transfer to neck strap muscles. Ann Otol Rhinol Laryngol. 113(9), 754-764, 2004.

7. Stepp CE, Heaton JT, Rolland RG, Hillman RE: Neck and face surface electromyography for prosthetic voice control after total laryngectomy. IEEE Trans Neural Syst Rehabil Eng. 17(2), 146$155,2009$.

8. Kubert HL, Stepp CE, Zeitels SM, Gooey JE, Walsh MJ, Prakash SR, Hillman RE, Heaton JT: Electromyographic control of a hands-free electrolarynx using neck strap muscles. J Commun Disord. 42(3), 211-225, 2009.

9. Nagle KF, Heaton JT: Perceived naturalness of electrolaryngeal speech produced using sEMG-controlled vs. manual pitch modulation. Proc of the Annual Conference of the International Speech Communication Association, INTERSPEECH, San Francisco, pp. 238-242, 2016.

10. Nakamura K, Toda T, Saruwatari H, Shikano K: Speaking-aid systems using GMM-based voice conversion for electrolaryngeal speech. Speech Commun. 54(1), 134-146, 2012.

11. Chadha A, Savardekar B, Padhya J: Analysis of a modern voice morphing approach using Gaussian mixture models for laryngec- tomees. Int J Comput Appl. 49(21), 25-30, 2012.

12. Tanaka K, Toda T, Neubig G, Sakti S, Nakamura S: Direct F0 control of an electrolarynx based on statistical excitation feature prediction and its evaluation through simulation. INTERSPEECH, Singapore, pp. 31-35, 2014.

13. Doi H, Toda T, Nakamura K, Saruwatari H, Shikano K: Alaryngeal speech enhancement based on one-to-many eigenvoice conversion. IEEE Trans Audio Speech Lang Process. 22(1), 172183, 2014.

14. Tanaka K, Toda T, Neubig G, Sakti S, Nakamura S: An evaluation of excitation feature prediction in a hybrid approach to electrolaryngeal speech enhancement. Proc of the IEEE International Conference on Acoustics, Speech and Signal Processing, Florence, pp. 4488-4492, 2014.

15. Tanaka K, Toda T, Neubig G, Sakti S, Nakamura S: An enhanced electrolarynx with automatic fundamental frequency control based on statistical prediction. Proc of the 17th International ACM SIGACCESS Conference on Computers and Accessibility, Lisbon, pp. 435-436, 2015.

16. Liu H, Zhao Q, Wan M, Supin W: Enhancement of electrolarynx speech based on auditory masking. IEEE Trans Biomed Eng. 53(5), 865-874, 2006.

17. Liu H, Ng ML: Electrolarynx in voice rehabilitation. Auris Nasus Larynx. 34(3), 327-332, 2007.

18. Li S, Wan MX, Wang SP: Multi-band spectral subtraction method for electrolarynx speech enhancement. Algorithms. 2(1), 550564, 2009.

19. Bhat RM, Singh JB, Lehana PK: Investigations of the effect of nonlinearly generated excitations on the quality of the synthesized alaryngeal speech. Indian J Sci Technol. 10(18), 1-12, 2017.

20. Xiao K, Wang S, Wan M, Wu L: Radiated noise suppression for electrolarynx speech based on multiband time-domain amplitude modulation. IEEE/ACM Trans Audio Speech Lang Process. 26(9), 1585-1593. 2018.

21. Malathi P, Suresh DGR, Moorthi DM: Enhancement of electrolaryngeal speech using frequency auditory masking and GMM based voice conversion. Proc of the 4th International Conference on Advances in Electrical, Electronics, Information, Communication and Bio-Informatics, Tamilnadu, pp. 1-4, 2018.

22. Ifukube T: Design of the voice typewriter, pp. 22-24, CQ publisher, 1983.

23. Hei T, Tanaka Y, Mizumachi M, Nakatoh Y, Matsui K: Study of natural-voice-like vibration sound for electrolarynx. Proc of the 2nd International Conference on Intelligent Systems and Image Processing, Kitakyushu, pp. 159-162, 2014.

24. Boersma P, van Heuven V: Speak and unSpeak with Praat. Glot Int. 5(9-10), 341-347, 2001.

25. Morikawa M: [Acoustic measurement and calibration] Onkyokeisoku to kyaribreesyon (in Japanese). J Acoust Soc Jpn. 74(6), 351-356, 2020.

26. Imaizumi S: [Alaryngeal Voicing Methods and Their Inherent Voice Quality] Daiyohasseihou to sono seishitsu (in Japanese). Jpn J Logopedics Phoniatrics. 24, 204-210, 1983.

27. Nieboer GLJ, de Graaf T, Schutte HK: Esophageal voice quality judgements by means of the semantic differential. J Phonetics. 16(4), 417-436, 1988.

28. Dall R, Yamagishi J, King S: Rating naturalness in speech Syn- 
thesis: The effect of style and expectation. Proc of the International Conference on Speech Prosody, Dublin, pp. 1012-1016, 2014.

29. Kishimoto M, Toda T, Doi H, Sakti S, Nakamura S: Model training using parallel data with mismatched pause positions in statistical esophageal speech enhancement. Proc of the International Conference on Signal Processing, Kuala Lumpur, pp. 590-594, 2012.

30. Werner S, Hoffmann R: Pronunciation variant selection for spontaneous speech synthesis - A summary of experimental results. Proc of the International Conference on Speech Prosody, Dresden, pp. 857-860, 2006

31. Speech Resources Consortium (NII-SRC): ATR 503 sentences. Retrieved from http://research.nii.ac.jp/src/en/ATR503.html. Accessed on October 17, 2021.

32. Doi H: Augmented speech production beyond physical constraints using statistical voice conversion: Alaryngeal speech enhancement and singing voice quality control, Ph.D. dissertation, 2013. https://library.naist.jp/mylimedio/dllimedio/showpdf2. cgi/DLPDFR009998_P1-128, (Accessed on July 25, 2021).

33. Takeuchi M, Ahn J, Matsufuji K, Lee K, Ogasawara Y, Takaki K, Ifukube T, Yabu K, Takamichi S, Ueha R, Sekino M, Onodera H: Development of a hands-free electrolarynx that obtains a voice close to human using the LPC residual wave. The papers of technical meeting on magnetics, IEE Japan 2020(73-79), 7-12, 2020.

\section{Masaki TAKeUCHI}

Masaki TAKEUCHI received his master's degree in Engineering from the University of Tokyo, Japan, in 2021. Since 2021, he has been pursuing his doctoral degree in the Graduate school of Engineering at The University of Tokyo.

\section{Jaesol AнN}

Jaesol AHN graduated from Changwon Science High School in 2015. He is currently an undergraduate student in the Department of Electronic Information Engineering, Faculty of Engineering, the University of Tokyo.

\section{Kunhak LeE}

Kunhak LeE graduated from Yuseong High School in 2017. He is currently an undergraduate student in the Department of Mechnaical Engineering, Faculty of Engineering, the University of Tokyo. His research interest includes Millifluidics. He is going to be a member of HTSJ (The Heat Transfer Society of Japan) in April 2022

\section{Ken TAKAKI}

Ken TAKAKI received his master's degree in Engineering from the University of Tokyo, Japan, in 2021. Since 2021, he has been pursuing his doctoral degree in the Graduate school of Engineering at The University of Tokyo.

\section{Tohru IFUKuBE}

Tohru IfuKuBE received his master's degree from Hokkaido University, Japan, in 1971. From 1971 to 1989 , he worked as an assistant professor at the Research Institute of Applied Electricity, Hokkaido University, Japan. He was a visiting associate professor at Stanford University, Stanford, USA,

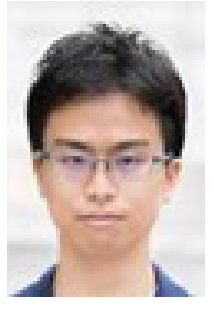
in 1984. From 1989 to 2002, he worked as a Professor at the Research Institute of Applied Electricity, Hokkaido University, Japan. From 2002 to 2007, he worked as a professor at the Research Center for Advanced Science and Technology (RCAST) at the University of Tokyo, Japan. From 2007 to 2009, he worked as an emeritus professor at Hokkaido University, Japan. From 2009 to 2011, he was an emeritus professor at the University of Tokyo, Japan. From 2011 to 2019, he was a Research Fellow at the Institute of Gerontology of the University of Tokyo, Japan. From 2015 to 2019, he worked as a professor at the Hokkaido University of Science, Japan. He has been an adviser at the RCAST, the University of Tokyo, Japan. His current research interests include assistive technology that supports the disabled and the elderly. He constructed a research methodology that circulates the basic science of psychophysiology and applied technologies, such as virtual reality and robots. He has led research on assistive devices for practical use.

\section{Ken-ichiro $\mathrm{Y}_{\mathrm{ABU}}$}

Ken-ichiro YABU received his Ph.D.(Engineering) degree from Tokyo Metropolitan University in 2010. From 2010 to 2014, he worked as a project researcher at the Research Center for Advanced Science and Technology, the University of Tokyo, Japan. Since 2014, he has been working as a proj-

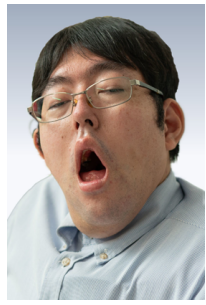
ect researcher at the Institute of Gerontology, the University of Tokyo. And since 2020, he has also been working as a project researcher at the Research Center for Advanced Science and Technology, the University of Tokyo. His research focuses on assistive device technology based on speech signal processing and information technologies and mechatronics systems for people with disabilities or the elderly. He is a member of the International Speech Communication Association (ISCA), Acoustical Society of Japan(ASJ), the Virtual Reality Society of Japan (VRSJ) etc. 


\section{Shinnosuke TAKAMICHI}

Shinnosuke TAKAмісні received his B.E. degree from Nagaoka University of Technology, Nagaoka, Japan, in 2011, and his M.E. and Ph.D. degrees from the Graduate School of Information Science, Nara Institute of Science and Technology, Ikoma, Japan, in 2013 and 2016, respectively.

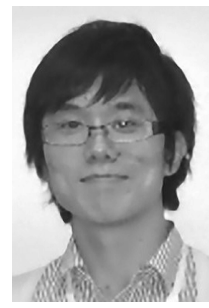

Since 2018, he has been working as an assistant professor at the University of Tokyo. He has received more than 20 papers/achievement awards, including the 2020 IEEE Signal Processing Society Young Author Best Paper Award.

\section{Rumi UeHA}

Rumi Ueha graduated from Nara Medical University, Faculty of Medicine, Japan, in 2003. After working as a resident at the University of Tokyo Hospital, she worked as an otolaryngologist at NTT East Kanto Hospital, Tokyo Metropolitan Neurological Hospital, and Kameda General Hos-

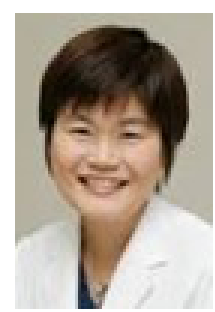
pital. From 2010 to 2012, she worked as a specially appointed clinician at the University of Tokyo Hospital, Japan. She was particularly interested in stroke, neurological intractable diseases, and dysphagia in patients with severe mental and physical disabilities. From 2012 to 2019, she worked as an assistant professor at the Department of Otorhinolaryngology, Head and Neck Surgery, University of Tokyo, Japan. She studied at the University of Michigan, USA, in 2012 and at the University of California, Davis, USA, in 2018. From 2019 to 2021, she was a project lecturer at the Department of Otorhinolaryngology, Head and Neck Surgery, University of Tokyo, Japan. Since 2021, she has been a director and associate professor at the Swallowing Center, University of Tokyo Hospital, Japan. Her specialization fields include swallowing disorders and olfactory impairment. She had undergone numerous surgeries and basic research. She is a member of the Association for Research in Otolaryngology.

\section{Masaki SeKINo}

Masaki SEKINO received his doctoral degree in Engineering from the University of Tokyo, Japan, in 2005. From 2005 to 2006, he worked as a project assistant at the Graduate School of Medicine, University of Tokyo, Japan. From 2006 to 2007, he worked as an assistant at the Graduate School of

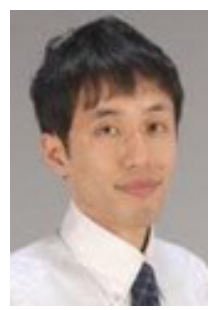
Frontier Sciences, University of Tokyo, Japan. From 2007 to 2010, he worked as an assistant professor at the Graduate School of Frontier Sciences, University of Tokyo, Japan. He was a French Government Scholarship student conducting research at the NeuroSpin Laboratory, CEA, France, in 2008. In 2009, he was an adjunct faculty member at the Bioimaging Center, Graduate School of Frontier Sciences, University of Tokyo, Japan. From 2010 to 2011, he worked as a lecturer at the Department of Electrical Engineering and Information Systems, University of Tokyo, Japan. From 2011 to 2020, he worked as an associate professor at the Department of Electrical Engineering and Information Systems, University of Tokyo, Japan. Since 2021, he has been working as a professor at the Department of Bioengineering, University of Tokyo, Japan. His current research interests include electromagnetic imaging of biological bodies and brain stimulation. He is a member of the Institute of Electrical and Electronics Engineers (IEEE), Bioelectromagnetics Society (BEMS), and International Society for Magnetic Resonance in Medicine (ISMRM). 